Document downloaded from:

http://hdl.handle.net/10251/61397

This paper must be cited as:

Calabuig Rodriguez, JM.; Fernandez Unzueta, M.; Galaz Fontes, F.; Sánchez Pérez, EA. (2014). Extending and factorizing bounded bilinear maps defined on order continuous Banach function spaces. Revista de la Real Academia de Ciencias Exactas, Físicas y Naturales. Serie A: Matemáticas (RACSAM). 108(2):353-367. doi:10.1007/s13398-0120101-7.

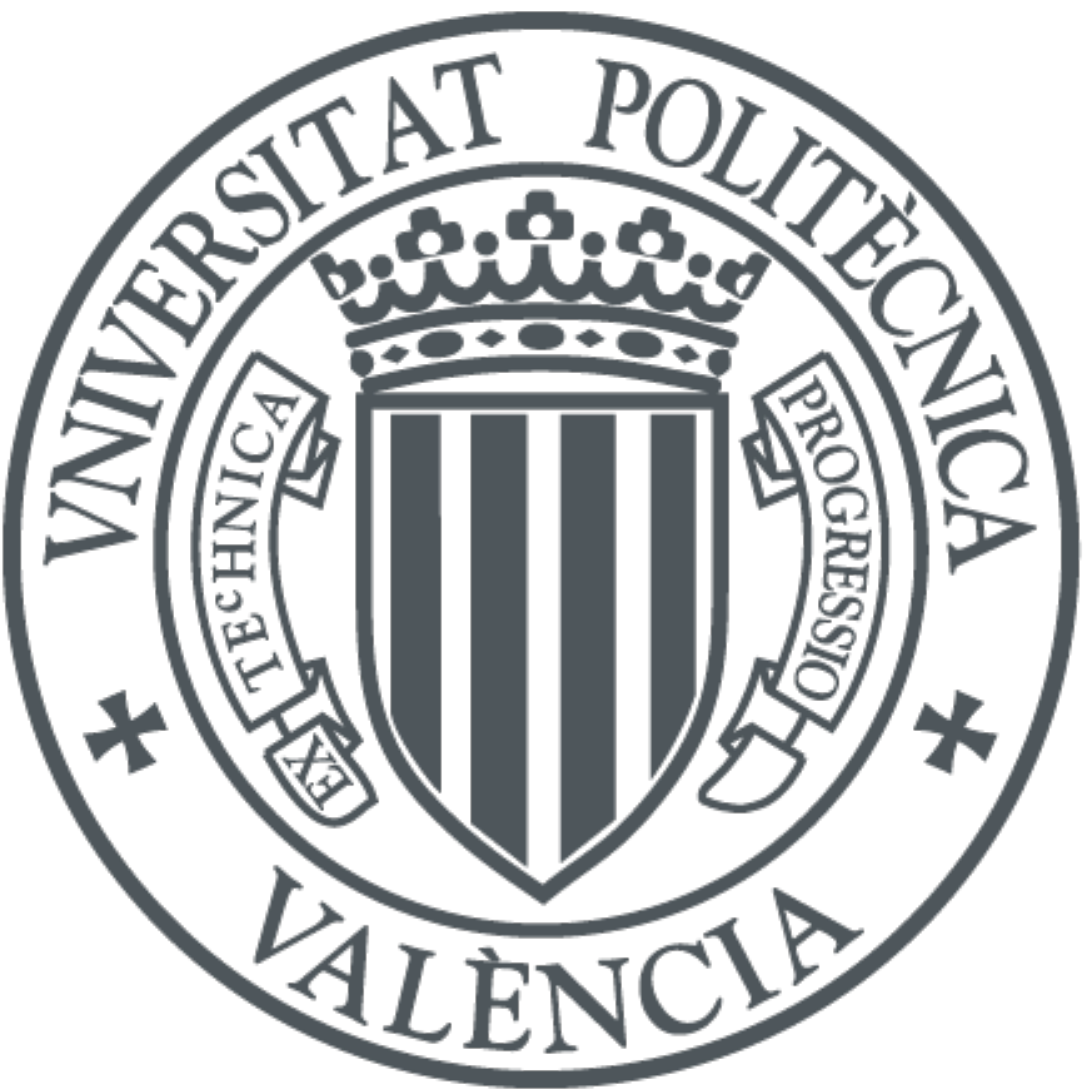

The final publication is available at

http://dx.doi.org/10.1007/s13398-012-0101-7

Copyright Springer

Additional Information 


\title{
EXTENDING AND FACTORIZING BOUNDED BILINEAR MAPS DEFINED ON ORDER CONTINUOUS BANACH FUNCTION SPACES
}

\author{
J.M. CALABUiG, M. FERNÁNDEZ UNZUETA, F. GALAZ-FONTES, \\ AND E.A. SÁNCHEZ-PÉREZ
}

\begin{abstract}
We consider the problem of extending or factorizing a bounded bilinear map defined on a couple of order continuous Banach function spaces to its optimal domain, i.e. the biggest couple of Banach function spaces to which the bilinear map can be extended. As in the case of linear operators, we use vector measure techniques to find this space, and we show that this procedure cannot be always successfully used for bilinear maps. We also present some applications to find optimal factorizations of linear operators between Banach function spaces.
\end{abstract}

\section{INTRODUCTION}

Let $X(\mu)$ be an order continuous Banach function space on the measure space $(\Omega, \Sigma, \mu)$, containing the set of all the characteristic functions. Take $E$ to be a Banach space, and consider a (linear and bounded) operator $T: X(\mu) \rightarrow E$, with associated vector measure $m_{T}$, that is, $m_{T}(A):=T\left(\chi_{A}\right), A \in \Sigma$. Then it is known that it is possible to factorize the operator $T$ through the space $L^{1}\left(m_{T}\right)$ of integrable functions with respect to $m_{T}$, i.e., the following diagram commutes

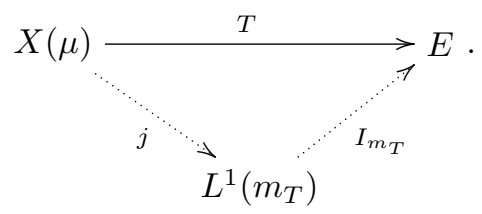

Here, $I_{m_{T}}$ is the integration operator and $j$ is a natural operator that coincides with the inclusion map $i$ in case any $m_{T}$-null set has $\mu$-measure zero. In this case, $T$ is

2000 Mathematics Subject Classification. 46E30, 46G10.

Key words and phrases. Order continuous Banach function spaces, vector measures, integrable functions, optimal domains, bilinear map.

J.M. Calabuig was supported by MEC and FEDER (project MTM2008-04594), by Generalitat Valenciana (project GV/2009/102) and by "Jose Castillejo 2009" (MEC). E.A. Sánchez-Pérez was supported by MEC and FEDER (project MTM2009-14483-C02-02). J.M. Calabuig and E.A. Sánchez-Pérez were also supported by "Ayuda para Estancias de PDI de la UPV en Centros de Investigación de Prestigio (PAID-00-11). 
called $\mu$-determined (see for instance [12, Ch.4], and [2] for non- $\mu$-determined operators). The space $L^{1}\left(m_{T}\right)$ that appears above is always optimal, in the sense that it contains any order continuous $\mu$-Banach function space to which the operator $T$ can be extended. This result -called the Optimal Domain Theorem for operators on Banach function spaces- has been used in a series of papers as a tool for describing the optimal domain for relevant operators, mainly in harmonic analysis (see for instance [4, 5, 8, 7, 12]).

Similar arguments have also been applied for obtaining the Optimal Domain of an operator that satisfies a particular domination property; the requirement is that the extension must also satisfy the same domination property. In this case, the optimal domain is given by a Banach function subspace of $L^{1}\left(m_{T}\right)$. Important examples of such construction has been recently obtained in [1, 12] and [2].

In this paper we study such kind of factorization through spaces of integrable functions in the case when we have a bounded bilinear map $\mathcal{B}: X(\mu) \times Y(\nu) \rightarrow Z$ instead of a linear one. In other words, we are interested in finding a factorization for $\mathcal{B}$ as

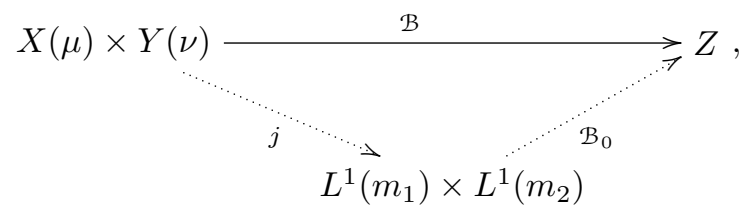

for suitable vector measures $m_{1}$ and $m_{2}$ and to analyze its optimality properties. Our first step is to use vector measures with values in the Banach space $L(Y, Z)$ of all operators going from the Banach space $Y$ to the Banach space $Z$. In order to factorize $\mathcal{B}$ we introduce the bounded linear map $S_{\mathcal{B}}: X(\mu) \rightarrow L(Y, Z)$ given by

$$
S_{\mathcal{B}}(f)(y)=\mathcal{B}(f, y) \text {, for all } f \in X(\mu) \text { and } y \in Y \text {. }
$$

As in the case of $\mu$-determined operators, the separation property

$$
\text { if } A \in \Sigma \text { and } \mu(A)>0 \text {, then } B\left(\chi_{C}, y\right) \neq 0 \text {, for some } C \in \Sigma, C \subset A, y \in Y \text {, }
$$

assures that any $m_{S_{\mathcal{B}}}$-null set is $\mu$-null and so the factorization obtained is indeed an extension. From the bounded linear map $S_{\mathcal{B}}: X(\mu) \rightarrow L(Y, Z)$ we obtain the space $L^{1}\left(m_{S_{\mathcal{B}}}\right)$, which provides the optimal extension of $S_{\mathcal{B}}$. If $\mathcal{B}$ does not have the property above, then the method we develop in Section 3 still gives a factorization for $\mathcal{B}$ through the product space $L^{1}\left(m_{S_{\mathcal{B}}}\right) \times Y(\mu)$ and, when $\mathcal{B}$ also has the corresponding property 3.2 , we obtain a factorization through a product space of the form $L^{1}\left(m_{1}\right) \times L^{1}\left(m_{2}\right)$. Theorem 3.4 establishes when this factorization is optimal.

On the other hand, given $y \in Y$ we can also consider the bounded linear map $S_{\mathcal{B}, y}: X(\mu) \rightarrow Z$ defined by

$$
S_{\mathcal{B}, y}(f)=\mathcal{B}(f, y)=S_{\mathcal{B}}(f)(y) \text {, for all } f \in X(\mu) .
$$


As before we can factorize each operator $S_{\mathcal{B}, y}$ through $L^{1}\left(m_{S_{\mathcal{B}, y}}\right)$. In Section 4 we relate the space of scalarly integrable functions $L_{w}^{1}\left(m_{S_{\mathcal{B}}}\right)$ with the spaces $L_{w}^{1}\left(m_{S_{\mathcal{B}, y}}\right)$, and the space $L^{1}\left(m_{S_{\mathcal{B}}}\right)$ with the spaces $L^{1}\left(m_{S_{\mathcal{B}, y}}\right)$, in order to give a description of our main factorization space $L^{1}\left(m_{S_{\mathcal{B}}}\right)$. Finally, we show in Section 5 some applications, providing under some restrictions the optimal factorization of an operator $T$ between Banach function spaces by applying our results to the bilinear form $\mathcal{B}(\cdot, \cdot):=\langle T(\cdot), \cdot\rangle$. Namely, our results can give the conditions under which it is possible to find a factorization diagram as

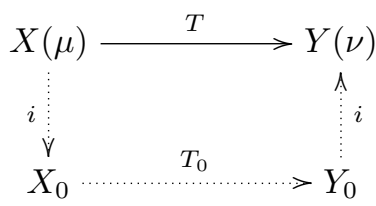

that extends in the left hand side to the biggest space in a class of B.f.s. and restricts the range to the smallest B.f.s. in other class of spaces.

\section{NotATION AND PRELIMINARIES}

Given a Banach space $E$ we denote by $E^{\prime}$ its topological dual and by $B_{E}$ its closed unit ball. By $\mathcal{P}(A)$ we will represent the set of partitions $\pi$ of $A \in \Sigma$, where $\pi$ has a finite number of disjoint measurable sets. If $1 \leq p \leq \infty$ then $p^{\prime} \in[1, \infty]$ is given by $1 / p+1 / p^{\prime}=1$.

Throughout this work $(\Omega, \Sigma, \mu)$ will always be a finite measure space. By $L^{0}(\Sigma)$ we will denote the space of all measurable real functions defined on $\Omega$ and by $L^{0}(\mu)$ the space of all equivalence classes of $\mu$-a.e. equal functions belonging to $L^{0}(\Sigma)$. We will call $\mu$-normed function space to any normed space $X(\mu) \subseteq L^{0}(\mu)$ having a lattice norm $\|\cdot\|_{X(\mu)}$ with respect to the $\mu$-a.e. natural order, that is, if $f \in L^{0}(\mu)$, $g \in X(\mu)$ and $|f| \leq|g| \mu$-a.e., then $f \in X(\mu)$ and $\|f\|_{X(\mu)} \leq\|g\|_{X(\mu)}$. Sometimes we write $X$ instead of $X(\mu)$ when the measure is clear in the context. When $X(\mu)$ is complete, we will say that $X(\mu)$ is a $\mu$-Banach function space ( $\mu$-B.f.s., for short). By a Banach function subspace of $X(\mu)$ we mean a $\mu$-B.f.s. continuously included in $X(\mu)$ (allowing different norms). A $\mu$-B.f.s. is order continuous if order bounded increasing sequences are convergent in norm. A $\mu$-normed function space $X(\mu)$ is said to have the Fatou property, if for any sequence $\left(f_{n}\right)_{n} \subset X(\mu)$ and $f \in L^{0}(\mu)$ such that $0 \leq f_{n} \uparrow f$ and $\left(\left\|f_{n}\right\|_{X(\mu)}\right)_{n}$ is bounded, we have that $f \in X(\mu)$ and $\left\|f_{n}\right\|_{X(\mu)} \uparrow\|f\|_{X(\mu)}$. Given two $\mu$-B.f.s. $X(\mu)$ and $Y(\mu)$ we will denote by $M(X, Y)$ the set of multipliers from $X(\mu)$ into $Y(\mu)$, that is, a function $h \in L^{0}(\mu)$ belongs to $M(X, Y)$ if (and only if) $h f \in Y(\mu)$ for all $f \in X(\mu)$. Under the adequate requirements, $M(X, Y)$ is again a Banach function space on $\mu$ when endowed with the operator norm. The product $X \cdot Y$ of two Banach function spaces $X(\mu)$ and 
$Y(\mu)$ is defined as the pairwise pointwise product of functions in each space, that is $X \cdot Y=\left\{h \in L^{0}(\Sigma): h=f g, f \in X(\mu), g \in Y(\mu)\right\}$.

Let $E$ be a Banach space and $m: \Sigma \rightarrow E$ be a (countably additive) vector measure. The semivariation of $m$ over $A \in \Sigma$ is defined by

$$
\|m\|(A)=\sup _{x^{\prime} \in B_{E^{\prime}}}\left|\left\langle m, x^{\prime}\right\rangle\right|(A),
$$

where $\left|\left\langle m, x^{\prime}\right\rangle\right|(A)$ is the variation of the scalar measure given by

$$
\left\langle m, x^{\prime}\right\rangle(A)=\left\langle m(A), x^{\prime}\right\rangle \text {, for each } A \in \Sigma .
$$

A set $A \in \Sigma$ is called $m$-null if $\|m\|(A)=0$. A property which holds outside an $m$-null set is said to hold $m$-almost everywhere (briefly, $m$-a.e.). A Rybakov measure for $m$ is a measure with the same null sets as $m$ and that has the form $\left|\left\langle m, x^{\prime}\right\rangle\right|$, where $x^{\prime} \in B_{E^{\prime}}$. It is well known that Rybakov measures always exist (see [9, IX.2.2]). Finally, we define $L^{0}(m):=L^{0}(\eta)$, where $\eta$ is a Rybakov measure for $m$.

Definition 2.1. A function $f: \Omega \rightarrow \mathbb{R}$ is said to be integrable with respect to the measure $m$ if

(a) $f$ is scalarly integrable with respect to $m$, that is, for each $x^{\prime} \in E^{\prime}$ we have that $f \in L^{1}\left(\left\langle m, x^{\prime}\right\rangle\right)$,

(b) for each $A \in \Sigma$ there exists $x_{A} \in E$ such that

$$
\left\langle x_{A}, x^{\prime}\right\rangle=\int_{A} f d\left\langle m, x^{\prime}\right\rangle \text { for every } x^{\prime} \in E^{\prime} .
$$

The vector $x_{A}$ is unique and will be denoted by $\int_{A} f d m$. The space of the classes with respect to equality $m$-a.e. of these functions is denoted by $L^{1}(m)$. The expression

$$
\|f\|_{m}=\sup _{x^{\prime} \in B_{E^{\prime}}} \int|f| d\left|\left\langle m, x^{\prime}\right\rangle\right| \text {, for each } f \in L^{1}(m),
$$

defines a lattice norm on $L^{1}(m)$, and so $L^{1}(m)$ is an order continuous $m$-B.f.s. (that is, it is an $\eta$-B.f.s., where $\eta$ is any Rybakov measure for the vector measure $m$; see for instance [3]). The indefinite integral $m_{f}: \Sigma \rightarrow E$ of a function $f \in L^{1}(m)$ is defined by

$$
m_{f}(A)=\int_{A} f d m, \quad A \in \Sigma .
$$

We will write $I_{m}: L^{1}(m) \rightarrow E$ for the integration map $I_{m}(f):=m_{f}(\Omega)$. For each integrable function $f$, the Orlicz-Pettis Theorem ensures that $m_{f}$ is again a countably additive vector measure. An equivalent norm for $L^{1}(m)$ is given by

$$
\|f\|_{m}=\sup _{A \in \Sigma}\left\|\int_{A} f d m\right\|_{E}, \text { for each } f \in L^{1}(m)
$$


which satisfies

$$
\|f\|_{m} \leq\|f\|_{m} \leq 2\|f\|_{m}
$$

(see [9, I.1.11]). The space $L_{w}^{1}(m)$ is the space of the classes (with respect to equality $m$-a.e.) of the scalarly integrable functions. The expression (2.1) also defines a norm in $L_{w}^{1}(m)$ and so we obtain again an $m$-B.f.s. with the Fatou property. Clearly $L^{1}(m)$ is a subspace of $L_{w}^{1}(m)$.

\section{Extending Bounded Bilinear Maps}

Let $X(\mu)$ be an order continuous $\mu$-B.f.s. and $Y$ and $Z$ be Banach spaces. We start this section by considering the problem of extending a given bounded bilinear map $\mathcal{B}: X(\mu) \times Y \rightarrow Z$. In order to do this we introduce the bounded linear map $S_{\mathcal{B}}: X(\mu) \rightarrow L(Y, Z)$ given by

$$
S_{\mathcal{B}}(f)(y)=\mathcal{B}(f, y) \text {, for all } f \in X(\mu) \text { and } y \in Y \text {. }
$$

We will also assume that $\mathcal{B}$ satisfies for every measurable set $A$

$$
\text { if } \mu(A)>0 \text {, then } \mathcal{B}\left(\chi_{C}, y\right) \neq 0 \text { for some } C \in \Sigma, C \subset A \text { and } y \in Y \text {. }
$$

This property assures that any $m_{S_{\mathcal{B}}}$-null set is $\mu$-null (see Lemma 4.6.

Applying the Optimal Domain Theorem for operators in Banach function spaces we can extend $S_{\mathcal{B}}$ to the space $L^{1}\left(m_{S_{\mathcal{B}}}\right)$. Hence the following diagram commutes

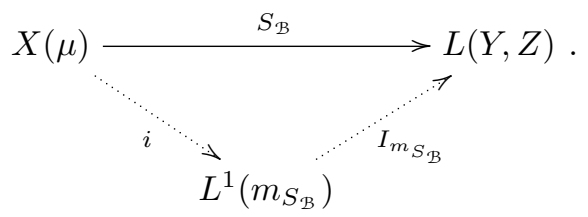

We now define $\mathcal{B}_{1}: L^{1}\left(m_{S_{\mathcal{B}}}\right) \times Y \rightarrow Z$ by

$$
\mathcal{B}_{1}(f, y)=\left(\int_{\Omega} f d m_{S_{\mathcal{B}}}\right)(y) .
$$

Proposition 3.1. i) The function $\mathcal{B}_{1}$ is a bilinear and bounded extension of $\mathcal{B}$.

ii) If $W(\mu)$ is an order continuous $\mu$-B.f.s. such that $X(\mu) \subset W(\mu), \mathcal{C}: W(\mu) \times$ $Y \rightarrow Z$ is bilinear and bounded and $\mathcal{C}=\mathcal{B}$ on $X(\mu) \times Y$, then $W(\mu) \subset L^{1}\left(m_{S_{\mathcal{B}}}\right)$ and $\mathcal{B}_{1}=\mathcal{C}$ on $W(\mu) \times Y$.

Proof. i) It is clear that $\mathcal{B}_{1}$ is bilinear. Let $f \in L^{1}\left(m_{S_{\mathcal{B}}}\right)$ and $y \in B_{Y}$. Then

$$
\left\|\mathcal{B}_{1}(f, y)\right\|_{Z}=\left\|\left(\int_{\Omega} f d m_{S_{B}}\right)(y)\right\|_{z} \leq\left\|\int_{\Omega} f d m_{S_{\mathcal{B}}}\right\|_{L(Y, Z)} \leq\|f\|_{L^{1}\left(m_{S_{\mathcal{B}}}\right)} .
$$

This shows that $\mathcal{B}_{1}$ is bounded.

ii) Let $W(\mu)$ and $\mathcal{C}$ be as in the hypothesis and consider the associated bounded linear operator $S_{\mathcal{e}}: W \rightarrow L(Y, Z)$. Since $X(\mu) \subset W(\mu)$ and $S_{\mathcal{e}}=S_{\mathcal{B}}$ on $X(\mu)$, by 
the Optimal Domain Theorem we have that $W(\mu) \subset L^{1}\left(m_{S_{\mathcal{B}}}\right)$ and $I_{m_{S_{\mathcal{B}}}}=S_{\mathcal{C}}$ on $W$. It follows that $\mathcal{B}_{1}=\mathcal{C}$ on $W(\mu) \times Y$.

In the above result we have fixed the second space, $Y$, and in doing so we were able to apply the methods of the linear case. We now discuss the possibility of varying $X$ or $Y$. Hence we consider both $X(\mu)$ and $Y(\mu)$ to be order continuous $\mu$-B.f.s. We consider the same measure $\mu$ for both spaces for the aim of simplicity, but the following construction also makes sense for a couple of Banach function spaces $X(\mu)$ and $Y(\nu)$ on different measures. Take $\mathcal{B}: X(\mu) \times Y(\mu) \rightarrow Z$ to be a bounded bilinear map having property 3.2 and also the following symmetric property; for each measurable set $A$,

$$
\text { if } \mu(A)>0 \text {, then } B\left(f, \chi_{C}\right) \neq 0 \text { for some } C \in \Sigma, C \subset A \text { and } f \in X \text {. }
$$

Applying the method we have introduced to the bounded linear map $T_{1}: X(\mu) \rightarrow$ $L(Y(\mu), Z)$ given by $T_{1}(f)=\mathcal{B}(f, \cdot)$, we find a vector valued measure $m_{1}: \Sigma \rightarrow$ $L(Y(\mu), Z)$ such that the following diagram commutes,

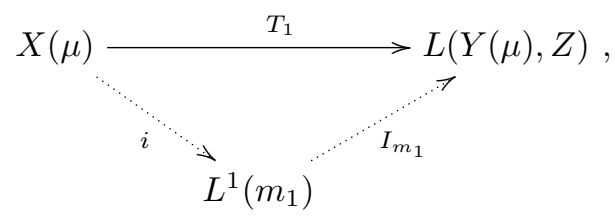

where $i$ is an inclusion map. Next we consider the bounded bilinear map $\mathcal{B}_{1}$ : $L^{1}\left(m_{1}\right) \times Y(\mu) \rightarrow Z$ as defined in 3.3 .

We can also apply the procedure explained above by fixing the first space factor instead of the second one. So we factorize now the operator $T_{12}: Y(\mu) \rightarrow$ $L\left(L^{1}\left(m_{1}\right), Z\right)$ given by $T_{12}(g)=\mathcal{B}_{1}(\cdot, g)$, obtaining a vector valued measure $\widetilde{m}_{2}$ : $\Sigma \rightarrow L\left(L^{1}\left(m_{1}\right), Z\right)$ for which the following diagram commutes,

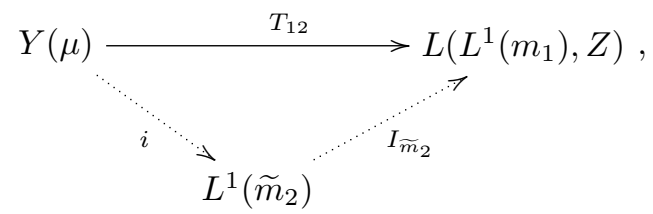

where $i$ an inclusion map. Let us take now the bounded bilinear map $\mathcal{B}_{12}: L^{1}\left(m_{1}\right) \times$ $L^{1}\left(\widetilde{m}_{2}\right) \rightarrow Z$ given by $\mathcal{B}_{12}(f, g)=\left(\int_{\Omega} g d \widetilde{m}_{2}\right)(f)$. Hence we have that the following diagram conmutes

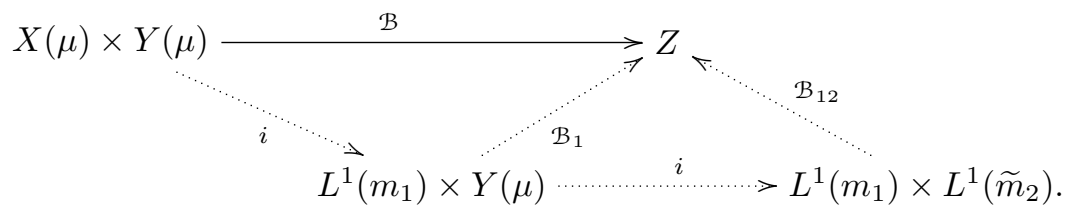


In a similar way we can find two vector valued measures $m_{2}: \Sigma \rightarrow L(X(\mu), Z)$ and $\widetilde{m}_{1}: \Sigma \rightarrow L\left(L^{1}\left(m_{2}\right), Z\right)$ satisfying that the following diagram conmutes

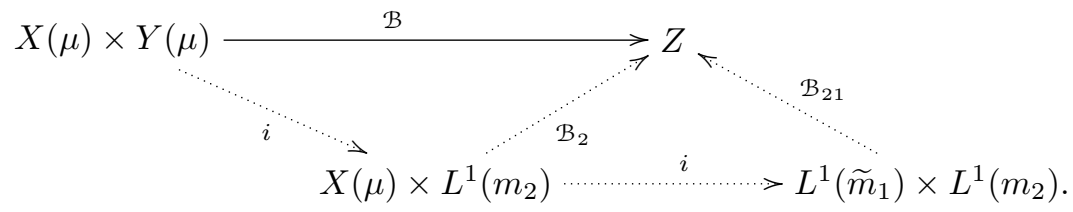

Therefore, we obtain two "non symmetric" factorizations in a direct way. The natural question that arises is: Is there an optimal factorization space?, if the answer is positive, then the candidate is of course $L^{1}\left(m_{1}\right) \times L^{1}\left(m_{2}\right)$. The following result shows that $\mathcal{B}$ can be extended bilinearly and continuously at most to the product space $L^{1}\left(m_{1}\right) \times L^{1}\left(m_{2}\right)$, although this factorization does not hold in general.

Proposition 3.2. If $\widetilde{X}(\mu)$ and $\widetilde{Y}(\mu)$ are order continuous Banach function spaces such that:

- $X(\mu) \times Y(\mu) \subseteq \tilde{X}(\mu) \times \tilde{Y}(\mu)$,

- there is a bounded bilinear map $\widetilde{\mathcal{J}}: \widetilde{X}(\mu) \times \widetilde{Y}(\mu) \rightarrow Z$ extending $\mathcal{B}$, then $\tilde{X}(\mu) \subseteq L^{1}\left(m_{1}\right)$ and $\tilde{Y}(\mu) \subseteq L^{1}\left(m_{2}\right)$.

Proof. We preserve the notation used aboved. Take $S: \widetilde{X}(\mu) \rightarrow L(Y(\mu), Z)$ given by $S(f)=\widetilde{\mathcal{J}}(f, \cdot)$. Take $f \in X(\mu)$ and $g \in Y(\mu)$. Then

$$
S(f)(g)=\widetilde{\mathcal{J}}(f, g)=\mathcal{B}(f, g)=T_{1}(f)(g) .
$$

Hence $T_{1}$ is the restriction of $S$ to $X(\mu)$ and thus the optimality of $L^{1}\left(m_{1}\right)$ in diagram 3.5 gives $\widetilde{X}(\mu) \subseteq L^{1}\left(m_{1}\right)$. In a similar way we obtain that $\widetilde{Y}(\mu) \subseteq$ $L^{1}\left(m_{2}\right)$.

Next, we show that it may not be possible to extend $\mathcal{B}$ to the cartesian product $L^{1}\left(m_{1}\right) \times L^{1}\left(m_{2}\right)$.

Example 3.3. Let $\lambda$ be the Lebesgue measure on the interval $[0,1]$ and consider the order continuous $\lambda$-B.f.s. $X(\lambda)=L^{2}[0,1]$ and $Y(\lambda)=L^{3}[0,1]$ and the Banach space $Z=L^{1}[0,1]$. Consider the bounded bilinear map

$$
\mathcal{B}_{0}: L^{2}[0,1] \times L^{3}[0,1] \rightarrow L^{1}[0,1], \quad \mathcal{B}_{0}(f, g)=f g .
$$

Notice $\mathcal{B}_{0}$ has properties $(3.2)$ and (3.4). Using that the space of multipliers $M\left(L^{p}[0,1], L^{1}[0,1]\right)$ equals $L^{p^{\prime}}[0,1]$ for all $1<p<\infty$ (see for instance [12, Propositions $3.43,3.66])$, we have

$$
L^{1}\left(m_{1}\right)=M\left(L^{3}[0,1], L^{1}[0,1]\right)=L^{3 / 2}[0,1] .
$$

On the other hand

$$
L^{1}\left(m_{2}\right)=M\left(L^{2}[0,1], L^{1}[0,1]\right)=L^{2}[0,1] .
$$


Notice that the pointwise product $L^{1}\left(m_{1}\right) \cdot L^{1}\left(m_{2}\right)$ gives $L^{3 / 2}[0,1] \cdot L^{2}[0,1]=$ $L^{6 / 7}[0,1]$ and $L^{1}[0,1] \subsetneq L^{6 / 7}[0,1]$, we can find functions $f \in L^{1}\left(m_{1}\right)$ and $g \in$ $L^{1}\left(m_{2}\right)$ such that $f g \notin L^{1}[0,1]$. Thus $\mathcal{B}_{0}$ cannot be extended to $L^{1}\left(m_{1}\right) \times L^{1}\left(m_{2}\right)$.

In our next result we characterize when the bilinear map $\mathcal{B}$ can be extended to the cartesian product $L^{1}\left(m_{1}\right) \times L^{1}\left(m_{2}\right)$.

Theorem 3.4. The following assertions are equivalent.

(1) The bilinear map $\mathcal{B}$ can be extended continuously and as a bilinear map $\mathcal{J}$ to the cartesian product $L^{1}\left(m_{1}\right) \times L^{1}\left(m_{2}\right)$.

(2) The equalities $L^{1}\left(m_{1}\right)=L^{1}\left(\widetilde{m}_{1}\right)$ and $L^{1}\left(m_{2}\right)=L^{1}\left(\widetilde{m}_{2}\right)$ hold.

(3) The bounded bilinear maps $\mathcal{B}_{12}$ and $\mathcal{B}_{21}$ coincide.

Proof. Let us start by showing that (1) implies (2). We have established in (3.8) that $\mathcal{B}$ has a bilinear and continuous extension to $L^{1}\left(\widetilde{m}_{1}\right) \times L^{1}\left(m_{2}\right)$. By Proposition 3.2 this implies that $L^{1}\left(\widetilde{m}_{1}\right) \subseteq L^{1}\left(m_{1}\right)$.

We will show now that $L^{1}\left(m_{2}\right) \subseteq L^{1}\left(\widetilde{m}_{2}\right)$. Take the operator $S: L^{1}\left(m_{2}\right) \rightarrow$ $L\left(L^{1}\left(m_{1}\right), Z\right)$ given by $S(g)=\mathcal{J}(\cdot, g)$. For each $g \in Y(\mu)$ and $A \in \Sigma$ we have that

$$
T_{12}(g)\left(\chi_{A}\right)=\mathcal{B}\left(\chi_{A}, g\right)=S(g)\left(\chi_{A}\right)
$$

so $T_{12}(g)(\phi)=S(g)(\phi)$ for all $g \in Y(\mu)$ and each simple function $\phi$. Hence the density of the simple functions in $L^{1}\left(m_{1}\right)$ gives that $T_{12}(g)=S(g)$ for all $g \in$ $Y(\mu)$. Therefore $T_{12}$ is the restriction of $S$ to $Y(\mu)$ so the optimality of diagram (3.6) provides that $L^{1}\left(m_{2}\right) \subseteq L^{1}\left(\widetilde{m}_{2}\right)$. Proceeding in a similar way we prove that $L^{1}\left(m_{1}\right) \subseteq L^{1}\left(\widetilde{m}_{1}\right)$ and $L^{1}\left(\widetilde{m}_{2}\right) \subseteq L^{1}\left(m_{2}\right)$, which gives $(2)$.

We continue by proving that (2) implies (3). Note that (2) implies that the domains of $\mathcal{B}_{12}$ and $\mathcal{B}_{21}$ coincide. On the other hand since for all $A, C \in \Sigma$ we have that $\mathcal{B}_{12}\left(\chi_{A}, \chi_{C}\right)=\mathcal{B}\left(\chi_{A}, \chi_{C}\right)=\mathcal{B}_{21}\left(\chi_{A}, \chi_{C}\right)$ then again the density of the simple functions both in $L^{1}\left(m_{1}\right)$ and $L^{1}\left(m_{2}\right)$ together with the continuity of $\mathcal{B}$ give (3).

If we assume now (3) then just take $\mathcal{J}=\mathcal{B}_{12}=\mathcal{B}_{21}$ and consider the scheme (3.7) -or (3.8) - to obtain (1).

\section{The SPACES $\mathcal{E}_{w}\left(m_{S_{\mathcal{B}}}\right)$ AND $\mathcal{E}\left(m_{S_{\mathcal{B}}}\right)$}

In this section we provide a description of our main factorization space $L^{1}\left(m_{S_{\mathcal{B}}}\right)$. Let us return to the beginning of Section 3 and consider an order continuous $\mu$-B.f.s. $X(\mu)$, Banach spaces $Y$ and $Z$ and a bounded bilinear map $\mathcal{B}: X(\mu) \times Y \rightarrow Z$ with property (3.4). Then, for the bounded linear map $S_{\mathcal{B}}: X(\mu) \rightarrow L(Y, Z)$ defined in 3.1 we obtained a factorization through the space $L^{1}\left(m_{S_{\mathcal{B}}}\right)$. We proceed in this section in a different way. For each $y \in Y$, we can also consider the bounded linear $\operatorname{map} S_{\mathcal{B}, y}: X(\mu) \rightarrow Z$ given by

$$
S_{\mathcal{B}, y}(f)=\mathcal{B}(f, y)=S_{\mathcal{B}}(f)(y), \text { for all } f \in X(\mu) .
$$


As in Section 3 we can factorize each operator $S_{\mathcal{B}, y}$ through the space $L^{1}\left(m_{S_{\mathcal{B}, y}}\right)$ as

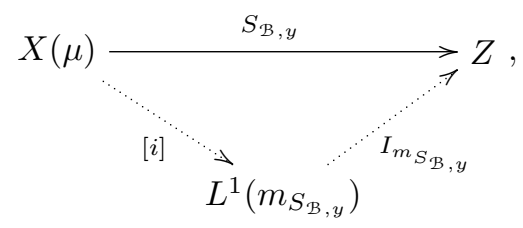

where $[i]$ is the (continuous) inclusion/quotient map that identifies each function in $X(\mu)$ with it class of $m_{S_{\mathcal{B}, y}}$-a.e. equal functions. This map is not necessarily injective; the reader can find more information on these operators in [11. In this section we will relate the space $L_{w}^{1}\left(m_{S_{\mathcal{B}}}\right)$ with the spaces $L_{w}^{1}\left(m_{S_{\mathcal{B}, y}}\right)$, and the space $L^{1}\left(m_{S_{\mathcal{B}}}\right)$ with the spaces $L^{1}\left(m_{S_{\mathcal{B}, y}}\right)$. Our aim is to give a description of the space $L^{1}\left(m_{S_{\mathcal{B}}}\right)$ that was shown to be central in Section 3. Our first goal is to prove that $L_{w}^{1}\left(m_{S_{\mathcal{B}}}\right)=\mathcal{E}_{w}\left(m_{S_{\mathcal{B}}}\right)$, where

$$
\mathcal{E}_{w}\left(m_{S_{\mathcal{B}}}\right)=\left\{f \in L^{0}\left(m_{S_{\mathcal{B}}}\right): f \in L_{w}^{1}\left(m_{S_{\mathcal{B}, y}}\right) \text {, for all } y \in Y\right\} .
$$

Recall that the separation property given in 3.4 is assumed for $\mathcal{B}$. Let $f \in$ $L_{w}^{1}\left(m_{S_{\mathcal{B}}}\right)$ and $y \in Y$. For each $z^{\prime} \in Z^{\prime}$ we can consider the bounded linear map

$$
H_{y, z^{\prime}}: L(Y, Z) \rightarrow \mathbb{R}, \quad H_{y, z^{\prime}}(T)=\left\langle T(y), z^{\prime}\right\rangle .
$$

Clearly

$$
\left\langle m_{S_{\mathcal{B}, y}}, z^{\prime}\right\rangle=\left\langle m_{S_{\mathcal{B}}}, H_{y, z^{\prime}}\right\rangle,
$$

and if $y \in B_{Y}$ and $z^{\prime} \in B_{Z^{\prime}}$, then $H_{y, z^{\prime}} \in B_{L(Y, Z)^{\prime}}$. Hence, for $y \in B_{Y}$ we have

$$
\|f\|_{m_{S_{\mathcal{B}, y}}}=\sup _{z^{\prime} \in B_{Z^{\prime}}} \int_{\Omega}|f| d\left|\left\langle m_{S_{\mathcal{B}, y}}, z^{\prime}\right\rangle\right|=\sup _{z^{\prime} \in B_{Z^{\prime}}} \int_{\Omega}|f| d\left|\left\langle m_{S_{\mathcal{B}}}, H_{y, z^{\prime}}\right\rangle\right| \leq\|f\|_{m_{S_{\mathcal{B}}}}<\infty .
$$

It follows that

$$
L_{w}^{1}\left(m_{S_{\mathcal{B}}}\right) \subseteq \mathcal{E}_{w}\left(m_{S_{\mathcal{B}}}\right) \text { and } \sup _{y \in B_{Y}}\|f\|_{m_{S_{\mathcal{B}, y}}} \leq\|f\|_{m_{S_{\mathcal{B}}}} \text {, for all } f \in L_{w}^{1}\left(m_{S_{\mathcal{B}}}\right) .
$$

Motivated by 4.2 , we define

$$
\|f\|_{\mathcal{E}_{w}\left(m_{S_{\mathcal{B}}}\right)}=\sup _{y \in B_{Y}}\|f\|_{m_{S_{\mathcal{B}, y}}}, f \in \mathcal{E}_{w}\left(m_{S_{\mathcal{B}}}\right) .
$$

In our next results we assume a requirement for the bilinear map that allows to assure that the function 4.3 takes real values and defines a norm on $\varepsilon_{w}\left(m_{S_{\mathcal{B}}}\right)$.

Definition 4.1. A map $\mathcal{B}: X(\mu) \times Y \rightarrow Z$ is said to be right order bounded if:

(a) $X(\mu)$ is an order continuous $\mu$-B.f.s. and $Y$ and $Z$ are Banach lattices,

(b) $\mathcal{B}$ is bilinear and bounded, and

(c) $\mathcal{B}\left(\chi_{A}, y_{1}\right) \leq \mathcal{B}\left(\chi_{A}, y_{2}\right)$ for all $A \in \Sigma$ and $y_{1} \leq y_{2} \in Y$. 
Easy examples of right order bounded bilinear maps are the positive bilinear maps between Banach lattices. Indeed, when $X$ and $Y$ are Banach lattices then the cartesian product $X \times Y$ is also a Banach lattice with the norm $\|(x, y)\|_{X \times Y}=$ $\max \left(\|x\|_{X},\|y\|_{Y}\right)$ and the order $\left(x_{1}, y_{1}\right) \leq\left(x_{2}, y_{2}\right)$ if and only if $x_{1} \leq x_{2}$ and $y_{1} \leq y_{2}$. Take now a Banach lattice $Z$. A bilinear map $\mathcal{B}: X \times Y \rightarrow Z$ is said to be positive if

$$
0 \leq \mathcal{B}\left(x_{1}, y_{1}\right) \leq \mathcal{B}\left(x_{2}, y_{2}\right), \text { for all } 0 \leq\left(x_{1}, y_{1}\right) \leq\left(x_{2}, y_{2}\right) \in X \times Y .
$$

It is easy to check that since $\mathcal{B}$ is positive then

$$
|\mathcal{B}(x, y)| \leq \mathcal{B}(|(x, y)|),
$$

for all $(x, y) \in X \times Y$. In the other hand it is well-known that positive linear maps between Banach lattices are always bounded (see, for instance [10]). A similar argument allows us to prove that the same result is also true for bilinear maps.

Lemma 4.2. Let $X, Y$ and $Z$ be Banach lattices and let $\mathcal{B}: X \times Y \rightarrow Z$ a positive bilinear map. Then $\mathcal{B}$ is continuous.

Proof. Assume that $\mathcal{B}$ is not continuous. Then there exist $\left(x_{n}, y_{n}\right)_{n} \in X \times Y$ and $\delta>0$ satisfying that for all $n \in \mathbb{N}$

$$
\left\|\left(x_{n}, y_{n}\right)\right\|_{X \times Y}<\frac{1}{2^{n}} \quad \text { and } \quad\left\|\mathcal{B}\left(x_{n}, y_{n}\right)\right\|_{Z}>\delta .
$$

Note that the series $\sum_{i \geq 1} i\left|\left(x_{i}, y_{i}\right)\right|$ is convergent in $X \times Y$, since for all $p \in \mathbb{N}$ one has

$$
\left\|\sum_{i=n}^{p} i\left|\left(x_{i}, y_{i}\right)\right|\right\|_{X \times Y} \leq \sum_{i=n}^{p} i\left\|\left(x_{i}, y_{i}\right)\right\|_{X \times Y} \leq \sum_{i=n}^{p} \frac{i}{2^{i}} .
$$

Denote $(a, b)=\sum_{i \geq 1} i\left|\left(x_{i}, y_{i}\right)\right| \in X \times Y$. Since $0 \leq n\left|\left(x_{n}, y_{n}\right)\right| \leq(a, b)$ and $\mathcal{B}$ is positive, we have $0 \leq \mathcal{B}\left(n\left|\left(x_{n}, y_{n}\right)\right|\right) \leq \mathcal{B}(a, b)$. Then, using (4.4),

$$
Z \ni \mathcal{B}(a, b) \geq \mathcal{B}\left(n\left|\left(x_{n}, y_{n}\right)\right|\right)=n^{2} \mathcal{B}\left(\left|\left(x_{n}, y_{n}\right)\right|\right) \geq n^{2}\left|\mathcal{B}\left(x_{n}, y_{n}\right)\right|,
$$

and so

$$
\|\mathcal{B}(a, b)\|_{Z} \geq n^{2}\left\|\mathcal{B}\left(x_{n}, y_{n}\right)\right\| \geq n^{2} \delta,
$$

for all $n \in \mathbb{N}$. This produces a contradiction, so $\mathcal{B}$ is continuous.

Example 4.3. Let $X(\mu)$ be an order continuous $\mu$-B.f.s. and let $Y$ and $Z$ be Banach lattices. Then every positive bilinear map $\mathcal{B}: X(\mu) \times Y \rightarrow Z$ is right order bounded.

Example 4.4. Take $X(\mu)$ and $Y(\mu)$ two $\mu$-B.f.s. such that the pointwise product $X(\mu) \cdot Y(\mu)$ is contained in $L^{1}(m)$ and consider the bilinear map $\mathcal{B}: X(\mu) \times Y(\mu) \rightarrow$ $L^{1}(m)$, given by $\mathcal{B}(f, g)=f g$, for all $f \in X(\mu)$ and $g \in Y(\mu)$. Then $\mathcal{B}$ is right order bounded. 
Let us show that, as we said before, for a $\mathcal{B}$ in the class of right order bounded bilinear maps, formula 4.3 defines a norm on $\mathcal{E}_{w}\left(m_{S_{\mathcal{B}}}\right)$.

Lemma 4.5. Let $\mathcal{B}$ be a right order bounded bilinear map. Then $\|f\|_{\mathcal{E}_{w}\left(m_{S_{\mathcal{B}}}\right)}<\infty$ for every function $f \in \mathcal{E}_{w}\left(m_{S_{\mathcal{B}}}\right)$.

Proof. Take $f \in \mathcal{E}_{w}\left(m_{S_{\mathcal{B}}}\right)$ and let us consider the function

$$
\mathcal{A}: Y \times Z^{\prime} \rightarrow[0, \infty), \quad \mathcal{A}\left(y, z^{\prime}\right)=\int_{\Omega}|f| d\left\langle m_{S_{B, y}}, z^{\prime}\right\rangle .
$$

Since $f \in \mathcal{E}_{w}\left(m_{S_{\mathcal{B}}}\right)$ then $\mathcal{A}$ is a well defined bilinear map that is separately continuous so continuous. Recall that, in particular, this means that $\|\mathcal{A}\|=$ $\sup _{y \in B_{Y}} \sup _{z^{\prime} \in B_{Z^{\prime}}}\left|\mathcal{A}\left(y, z^{\prime}\right)\right|$ must be finite. On the other hand condition (3) in the definition of right order bounded map gives that for all $A \in \Sigma, y \in Y$ and $z^{\prime} \in Z^{\prime}$

$$
\begin{aligned}
\left|\left\langle m_{S_{\mathcal{B}, y}}, z^{\prime}\right\rangle\right|(A) & =\sup _{\pi \in \mathcal{P}(A)} \sum_{C \in \pi}\left|\left\langle m_{S_{\mathcal{B}, y}}, z^{\prime}\right\rangle(C)\right|=\sup _{\pi \in \mathcal{P}(A)} \sum_{C \in \pi}\left|\left\langle\mathcal{B}\left(\chi_{C}, y\right), z^{\prime}\right\rangle\right| \\
& \leq \sup _{\pi \in \mathcal{P}(A)} \sum_{C \in \pi}\left\langle\left|\mathcal{B}\left(\chi_{C}, y\right)\right|,\left|z^{\prime}\right|\right\rangle \leq \sup _{\pi \in \mathcal{P}(A)} \sum_{C \in \pi}\left\langle\mathcal{B}\left(\chi_{C},|y|\right),\left|z^{\prime}\right|\right\rangle \\
& =\left\langle\mathcal{B}\left(\chi_{A},|y|\right),\left|z^{\prime}\right|\right\rangle=\left\langle m_{S_{\mathcal{B},|y|}},\left|z^{\prime}\right|\right\rangle(A) .
\end{aligned}
$$

Therefore

$$
\begin{aligned}
\|f\|_{\mathcal{E}_{w}\left(m_{S_{\mathcal{B}}}\right)} & =\sup _{y \in B_{Y}}\|f\|_{m_{S_{\mathcal{B}, y}}}=\sup _{y \in B_{Y}} \sup _{z^{\prime} \in B_{Z^{\prime}}} \int_{\Omega}|f| d\left|\left\langle m_{S_{\mathcal{B}, y}}, z^{\prime}\right\rangle\right| \\
& \leq \sup _{y \in B_{Y}} \sup _{z^{\prime} \in B_{Z^{\prime}}} \int_{\Omega}|f| d\left\langle m_{S_{\mathcal{B},|y|},}\left|z^{\prime}\right|\right\rangle=\sup _{y \in B_{Y}} \sup _{z^{\prime} \in B_{Z^{\prime}}} \mathcal{A}\left(|y|,\left|z^{\prime}\right|\right) \\
& \leq\|\mathcal{A}\|<\infty .
\end{aligned}
$$

Next, by simple calculations we obtain that, if $\phi$ is a simple function,

$$
\|\phi\|_{m_{S_{\mathcal{B}}}}=\sup _{y \in B_{Y}}\|\phi\|_{m_{S_{\mathcal{B}, y}}} .
$$

Lemma 4.6. For each $A \in \Sigma$ we have

$$
\sup _{y \in B_{Y}}\left\|m_{S_{\mathcal{B}, y}}\right\|(A) \leq\left\|m_{S_{\mathcal{B}}}\right\|(A) \leq 2 \sup _{y \in B_{Y}}\left\|m_{S_{\mathcal{B}, y}}\right\|(A) .
$$

Proof. For the first inequality just use 4.1). Indeed, for each $y \in B_{Y}$

$$
\begin{aligned}
\left\|m_{S_{\mathcal{B}, y}}\right\|(A) & =\sup _{z^{\prime} \in B_{Z^{\prime}}} \sup _{\pi \in \mathcal{P}(A)} \sum_{C \in \pi}\left|\left\langle m_{S_{\mathcal{B}, y}}(C), z^{\prime}\right\rangle\right| \\
& =\sup _{z^{\prime} \in B_{Z^{\prime}}} \sup _{\pi \in \mathcal{P}(A)} \sum_{C \in \pi}\left|\left\langle m_{S_{\mathcal{B}}}(C), H_{y, z^{\prime}}\right\rangle\right| \\
& \leq\left\|m_{S_{\mathcal{B}}}\right\|(A) .
\end{aligned}
$$


To establish the other inequality we use 2.2 and 4.5 to find that

$$
\left\|m_{S_{\mathcal{B}}}\right\|(A)=\left\|\chi_{A}\right\|_{m_{S_{\mathcal{B}}}} \leq 2\left\|\chi_{A}\right\|\left\|_{m_{S_{\mathcal{B}}}}=2 \sup _{y \in B_{Y}}\right\| \chi_{A}\|\|_{m_{S_{\mathcal{B}, y}}} \leq 2 \sup _{y \in B_{Y}}\left\|\chi_{A}\right\|_{m_{S_{\mathcal{B}, y}}} .
$$

Theorem 4.7. Let $\mathcal{B}$ be a right order bounded map. The function $\|\cdot\|_{\varepsilon_{w}\left(m_{S_{\mathcal{B}}}\right)}$ is a norm on the vector space $\mathcal{E}_{w}\left(m_{S_{\mathcal{B}}}\right)$. With this norm $\mathcal{E}_{w}\left(m_{S_{\mathcal{B}}}\right)$ is a $m_{S_{\mathcal{B}}}-$ B.f.s. with the Fatou property.

Proof. Since the remaining properties are clear, in order to see that the function $\|\cdot\|_{\mathcal{E}_{w}\left(m_{S_{\mathcal{B}}}\right)}$ defines a norm (with respect to $\left\|m_{S_{\mathcal{B}}}\right\|$-a.e. equality), we will only prove that $\|f\|_{\mathcal{E}_{w}\left(m_{S_{\mathcal{B}}}\right)}=0$ if and only if $f=0,\left\|m_{S_{\mathcal{B}}}\right\|$-a.e. If $f=0,\left\|m_{S_{\mathcal{B}}}\right\|$-a.e., then by using Lemma 4.6 we have that $\|f\|_{\mathcal{E}_{w}\left(m_{S_{\mathcal{B}}}\right)}=0$. For the reverse implication take $f \in \mathcal{E}_{w}\left(m_{S_{\mathcal{B}}}\right)$ with $\|f\|_{\mathcal{E}_{w}\left(m_{S_{\mathcal{B}}}\right)}=0$ and let $A=\{w \in \Omega: f(w) \neq 0\}$. Then $A \in \Sigma$ and $m_{S_{\mathcal{B}, y}}(A)=0$, for each $y \in B_{Y}$. By Lemma 4.6 this implies that $m_{\mathcal{B}}(A)=0$. Hence $|f|=0,\left\|m_{S_{\mathcal{B}}}\right\|$-a.e..

Since normed function spaces with the Fatou property are always complete, we only need to establish that $\mathcal{E}_{w}\left(m_{S_{\mathcal{B}}}\right)$ has this property. Take $\left(f_{n}\right)_{n} \subseteq \mathcal{E}_{w}\left(m_{S_{\mathcal{B}}}\right)$ and $0 \neq f \in L^{0}(\Sigma)$ such that $0 \leq f_{n} \uparrow|f|$ and $\left\|f_{n}\right\|_{\mathcal{E}_{w}\left(m_{S_{\mathcal{B}}}\right)} \leq k$ for all $n \in \mathbb{N}$. We

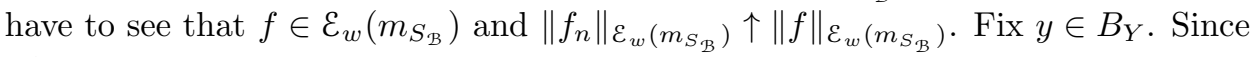
$L_{w}^{1}\left(m_{S_{\mathcal{B}, y}}\right)$ has the Fatou property and $\left\|f_{n}\right\|_{\mathcal{E}_{w}\left(m_{S_{\mathcal{B}}}\right)}=\sup _{y \in B_{Y}}\left\|f_{n}\right\|_{m_{S_{\mathcal{B}, y}}} \leq k$, then $f \in L_{w}^{1}\left(m_{S_{\mathcal{B}, y}}\right)$. Take now $0<r<\|f\|_{\varepsilon_{w}\left(m_{S_{\mathcal{B}}}\right)}$. Hence there is $y_{0} \in B_{Y}$

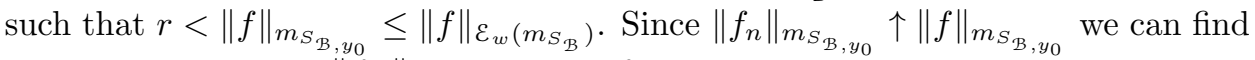
$N \in \mathbb{N}$ such that $r<\left\|f_{N}\right\|_{m_{S_{\mathcal{B}, y_{0}}}}$. Therefore

$$
\left\|f_{N}\right\|_{\mathcal{E}_{w}\left(m_{S_{\mathcal{B}}}\right)}=\sup _{y \in B_{Y}}\left\|f_{N}\right\|_{m_{S_{\mathcal{B}, y}}} \geq\left\|f_{N}\right\|_{m_{S_{\mathcal{B}, y_{0}}}}>r .
$$

It follows that $\left\|f_{n}\right\|_{\mathcal{E}_{w}\left(m_{S_{\mathcal{B}}}\right)} \uparrow\|f\|_{\varepsilon_{w}\left(m_{S_{\mathcal{B}}}\right)}$.

Corollary 4.8. Let $\mathcal{B}$ be a right order bounded map. The equality $\mathcal{E}_{w}\left(m_{S_{\mathcal{B}}}\right)=$ $L_{w}^{1}\left(m_{S_{\mathcal{B}}}\right)$ holds, and

$$
\|f\|_{\varepsilon_{w}\left(m_{S_{\mathcal{B}}}\right)} \leq\|f\|_{m_{S_{\mathcal{B}}}} \leq 2\|f\|_{\mathcal{E}_{w}\left(m_{S_{\mathcal{B}}}\right)}, f \in \mathcal{E}_{w}\left(m_{S_{\mathcal{B}}}\right) .
$$

Proof. By 4.2 it remains to prove that every function $f \in \mathcal{E}_{w}\left(m_{S_{\mathcal{B}}}\right)$ belongs to $L_{w}^{1}\left(m_{S_{\mathcal{B}}}\right)$ and to establish the inequality that appears on the right hand side of 4.6. Hence take $f \in \mathcal{E}_{w}\left(m_{S_{\mathcal{B}}}\right)$ and apply Theorem 4.7 to obtain a sequence $\left(\phi_{n}\right)_{n}$ of simple functions such that $0 \leq \phi_{n} \uparrow|f|$ and $\left\|\phi_{n}\right\|_{\mathcal{E}_{w}\left(m_{S_{\mathcal{B}}}\right)} \uparrow\|f\|_{\mathcal{E}_{w}\left(m_{S_{\mathcal{B}}}\right)}$. Using now 2.2 and 4.5 we obtain

$$
\begin{aligned}
\left\|\phi_{n}\right\|_{m_{S_{\mathcal{B}}}} & \leq 2\left\|\phi_{n}\right\|_{m_{S_{\mathcal{B}}}}=2 \sup _{y \in B_{Y}}\left\|\phi_{n}\right\|_{m_{S_{\mathcal{B}, y}}} \leq 2 \sup _{y \in B_{Y}}\left\|\phi_{n}\right\|_{m_{\mathcal{B}_{\mathcal{B}, y}}} \\
& =2\left\|\phi_{n}\right\|_{\mathcal{E}_{w}\left(m_{S_{\mathcal{B}}}\right)} \leq 2\|f\|_{\mathcal{E}_{w}\left(m_{S_{\mathcal{B}}}\right) .}
\end{aligned}
$$

Since $L_{w}^{1}\left(m_{S_{\mathcal{B}}}\right)$ has the Fatou property, then $f \in L_{w}^{1}\left(m_{S_{\mathcal{B}}}\right)$ and $\left\|\phi_{n}\right\|_{m_{S_{\mathcal{B}}}} \uparrow\|f\|_{m_{S_{\mathcal{B}}}}$. It follows now from 4.7 that $\|f\|_{m_{S_{\mathcal{B}}}} \leq 2\|f\|_{\mathcal{E}_{w}\left(m_{S_{\mathcal{B}}}\right)}$. 
Although the description of $L^{1}\left(m_{S_{\mathcal{B}}}\right)$ is given by $\varepsilon_{w}\left(m_{S_{\mathcal{B}}}\right)$ for the frequent cases when $L^{1}\left(m_{S_{\mathcal{B}}}\right)=L_{w}^{1}\left(m_{S_{\mathcal{B}}}\right)$-for instance, when $Z$ is a reflexive space, see Proposition 3.38 in 12 - it seems natural to consider now the space

$$
\mathcal{E}\left(m_{S_{\mathcal{B}}}\right)=\left\{f \in L^{0}(\Sigma): f \in L^{1}\left(m_{S_{\mathcal{B}, y}}\right), \text { for all } y \in Y\right\} \subseteq \mathcal{E}_{w}\left(m_{S_{\mathcal{B}}}\right),
$$

and try to relate it with $L^{1}\left(m_{S_{\mathcal{B}}}\right)$. We start by proving the completeness of this space endowed with the norm $\|\cdot\|_{\mathcal{E}_{w}\left(m_{S_{\mathcal{B}}}\right)}$.

Proposition 4.9. Let $\mathcal{B}$ be a right order bounded map. Then $\left(\mathcal{E}\left(m_{S_{\mathcal{B}}}\right),\|\cdot\|_{\mathcal{E}_{w}\left(m_{S_{\mathcal{B}}}\right)}\right)$ is an $m_{S_{\mathcal{B}}}$-Banach function space.

Proof. We only have to prove the completeness. Therefore take $\left(f_{n}\right)_{n}$, a Cauchy sequence in $\mathcal{E}\left(m_{S_{\mathcal{B}}}\right)$. Then, by Corollary $4.8\left(f_{n}\right)_{n}$ is also a Cauchy sequence in $L_{w}^{1}\left(m_{S_{\mathcal{B}}}\right)$. The completeness of this space allow us to find a function $f \in L_{w}^{1}\left(m_{S_{\mathcal{B}}}\right)$ such that $\left(f_{n}\right)_{n}$ converges to $f$ in $L_{w}^{1}\left(m_{S_{\mathcal{B}}}\right)$. Let us show that $f \in \mathcal{E}\left(m_{S_{\mathcal{B}}}\right)$. Indeed, fix $y \in Y$. By the definition of the norm $\|\cdot\|_{\mathcal{E}_{w}\left(m_{S_{\mathcal{B}}}\right)}$ the sequence $\left(f_{n}\right)_{n}$ converges to $f$ in $L_{w}^{1}\left(m_{S_{\mathcal{B}, y}}\right)$. But since $\left(f_{n}\right)_{n} \subseteq L^{1}\left(m_{S_{\mathcal{B}, y}}\right)$ and $L^{1}\left(m_{S_{\mathcal{B}, y}}\right)$ is closed in $L_{w}^{1}\left(m_{S_{\mathcal{B}, y}}\right)$ then $f \in L^{1}\left(m_{S_{\mathcal{B}, y}}\right)$. Therefore $f \in \mathcal{E}\left(m_{S_{\mathcal{B}}}\right)$.

Let us discuss now the equality $\mathcal{E}\left(m_{S_{\mathcal{B}}}\right)=L^{1}\left(m_{S_{\mathcal{B}}}\right)$. First of all note that for each $y \in B_{Y}$, since $L^{1}\left(m_{S_{\mathcal{B}}}\right)$ is a Banach function space that contains (continuously) $X(\mu)$, then the optimality of the factorization

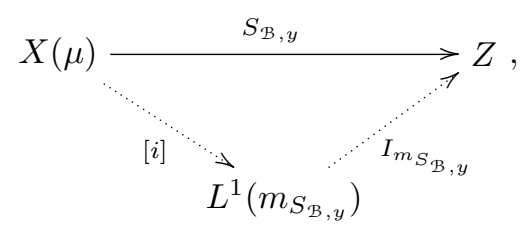

gives that $[i]\left(L^{1}\left(m_{S_{\mathcal{B}}}\right)\right) \subseteq L^{1}\left(m_{S_{\mathcal{B}, y}}\right)$ - where $[i]$ is the inclusion/quotient map that was explained in the Introduction, see [2, 11] - for all $y \in B_{Y}$. Therefore, always under the assumption that $\mathcal{B}$ is a right order bounded map, taking into account that $\mathcal{E}\left(m_{S_{\mathcal{B}}}\right)$ is an $m_{S_{\mathcal{B}}}$-B.f.s. and so the equivalence classes of functions in $L^{1}\left(m_{S_{\mathcal{B}}}\right)$ and $\mathcal{E}\left(m_{S_{\mathcal{B}}}\right)$ coincide, we obtain

$$
L^{1}\left(m_{S_{\mathcal{B}}}\right) \subseteq \mathcal{E}\left(m_{S_{\mathcal{B}}}\right) .
$$

The following simple characterization will be used to give an example where $L^{1}\left(m_{S_{\mathcal{B}}}\right) \subsetneq \mathcal{E}\left(m_{S_{\mathcal{B}}}\right)$.

Proposition 4.10. Let $\mathcal{B}$ be a right order bounded map. The following assertions are equivalent.

(a) $\mathcal{E}\left(m_{S_{\mathcal{B}}}\right)=L^{1}\left(m_{S_{\mathcal{B}}}\right)$,

(b) $\mathcal{E}\left(m_{S_{\mathcal{B}}}\right)$ is order continuous. 
Proof. Clearly (a) implies (b). For the converse take $f \in \mathcal{E}\left(m_{S_{\mathcal{B}}}\right), f \geq 0, m_{S_{\mathcal{B}}}$-a.e. and choose a sequence of simple function $\left(\phi_{n}\right)_{n}$ such that $0 \leq \phi_{n} \uparrow f$. Since we are assuming $\mathcal{E}\left(m_{S_{\mathcal{B}}}\right)$ is order continuous, it follows that $\phi_{n} \rightarrow f$ in $\mathcal{E}\left(m_{S_{\mathcal{B}}}\right)$. By Corollary 4.8 , this implies that $\left(\phi_{n}\right)_{n}$ is a Cauchy sequence in $L^{1}\left(m_{S_{\mathcal{B}}}\right)$. Hence, we have that $\phi_{n} \rightarrow g$ in $L^{1}\left(m_{S_{\mathcal{B}}}\right)$, for some $g \in L^{1}\left(m_{S_{\mathcal{B}}}\right)$. It follows that $f=g, m_{S_{\mathcal{B}}}$ a.e., and thus $f \in L^{1}\left(m_{S_{\mathcal{B}}}\right)$. This implies that $\mathcal{E}\left(m_{S_{\mathcal{B}}}\right) \subseteq L^{1}\left(m_{S_{\mathcal{B}}}\right)$.

Example 4.11. Let $1 \leq p<\infty$ and consider the positive (and in particular right order bounded) bilinear map

$$
\mathcal{B}: \ell^{1} \times \ell^{p} \rightarrow \ell^{p}, \quad \mathcal{B}(x, y)=x y .
$$

Fix $y \in \ell^{p}$. Hence, $m_{S_{\mathcal{B}, y}}(A)=y \chi_{A}$, for each $A \subseteq \mathbb{N}$. Then it is well-known (cf. [12, Corollary 3.66]) that

$$
L^{1}\left(m_{S_{\mathcal{B}, y}}\right)=\left\{x: x y \in \ell^{p}\right\} .
$$

Moreover, $\|x\|_{m_{S_{\mathcal{B}, y}}}=\|x y\|_{\ell^{p}}$, for all $x \in L^{1}\left(m_{S_{\mathcal{B}, y}}\right)$.

On the other hand since $M\left(\ell^{p}, \ell^{p}\right)=\ell^{\infty}$ (cf. [12, Lemma 2.80]), one has

$$
\mathcal{E}\left(m_{S_{\mathcal{B}}}\right)=\left\{x: x y \in \ell^{p}, \text { for all } y \in \ell^{p}\right\}=M\left(\ell^{p}, \ell^{p}\right)=\ell^{\infty} .
$$

Moreover

$$
\|x\|_{\mathcal{E}_{w}\left(m_{S_{\mathcal{B}}}\right)}=\sup _{y \in B_{\ell p}}\|x\|_{m_{S_{\mathcal{B}, y}}}=\sup _{y \in B_{\ell^{p}}}\|x y\|_{\ell^{p}}=\|x\|_{\ell^{\infty}} .
$$

Since $\mathcal{E}\left(m_{S_{\mathcal{B}}}\right)=\ell^{\infty}$ is not order continuous, then Proposition 4.10 together with (4.8) gives that $L^{1}\left(m_{S_{\mathcal{B}}}\right) \subsetneq \mathcal{E}\left(m_{S_{\mathcal{B}}}\right)$.

However, there are many cases where the equality $\mathcal{E}\left(m_{S_{\mathcal{B}}}\right)=L^{1}\left(m_{S_{\mathcal{B}}}\right)$ holds, as our next example shows.

Example 4.12. Let us consider a right order bounded map $\mathcal{B}: X(\mu) \times Y \rightarrow Z$, where $0<\operatorname{dim} Y<\infty$. We will show that $\mathcal{E}\left(m_{S_{\mathcal{B}}}\right)=L^{1}\left(m_{S_{\mathcal{B}}}\right)$.

Take a basis of $Y,\left\{y_{1}, \ldots, y_{n}\right\}$. Given $y \in B_{Y}$ then $y=\sum_{j=1}^{n} a_{j} y_{j}$. Clearly $m_{S_{\mathcal{B}, y}}=\sum_{j=1}^{n} m_{S_{\mathcal{B}, y_{j}}}$ and $\left|\left\langle m_{S_{\mathcal{B}, y}}, z^{\prime}\right\rangle\right| \leq \sum_{j=1}^{n}\left|a_{j}\right| \cdot\left|\left\langle m_{S_{\mathcal{B}, y_{j}}}, z^{\prime}\right\rangle\right|$ for all $z^{\prime} \in B_{Z^{\prime}}$. Therefore

$$
\sup _{y \in B_{Y}}\|f\|_{m_{S_{\mathcal{B}, y}}} \leq M \cdot \max _{1 \leq j \leq n}\|f\|_{m_{S_{\mathcal{B}, y_{j}}}},
$$

where $M=n \cdot \max \left\{\left|a_{j}\right|: 1 \leq j \leq n\right\}<\infty$. Consider now $f \in \mathcal{E}\left(m_{S_{\mathcal{B}}}\right)$. Hence there is a sequence $0 \leq\left(f_{n}\right)_{n} \subseteq L^{0}(\Sigma)$ such that $0 \leq f_{n} \uparrow|f|$. Since $f \in L^{1}\left(m_{S_{\mathcal{B}, y_{j}}}\right)$ for all $1 \leq j \leq n$ and $L^{1}\left(m_{S_{B, y_{j}}}\right)$ is order continuous then $f_{n} \rightarrow|f|$ in $L^{1}\left(m_{S_{\mathcal{B}, y_{j}}}\right)$ for each $1 \leq j \leq n$. By using (4.9) we conclude that $f_{n} \rightarrow|f|$ in $\mathcal{E}\left(m_{S_{\mathcal{B}}}\right)$ and therefore $\mathcal{E}\left(m_{S_{\mathcal{B}}}\right)$ is order continuous. So Proposition 4.10 gives the equality $\mathcal{E}\left(m_{S_{\mathcal{B}}}\right)=$ $L^{1}\left(m_{S_{\mathcal{B}}}\right)$. 


\section{Applications: Optimal factorizations for operators Between BANACH FUNCTION SPACES}

In this last section we apply the results presented in the previous ones to the particular case of finding - if possible - the optimal factorization for an operator between Banach function spaces. Let $T: X(\mu) \rightarrow Y(\mu)$ be an operator from an order continuous space $X(\mu)$ to a space $Y(\mu)$. In the Introduction we explained that in case $T$ is $\mu$-determined, factorization through the space $L^{1}\left(m_{T}\right)$ provides the optimal extension, i.e. the extension of $T$ to the biggest order continuous Banach function space. In [11, the same kind of arguments have been used to obtain what is called a factorization with an optimal range, i.e. finding a factorization for the operator as

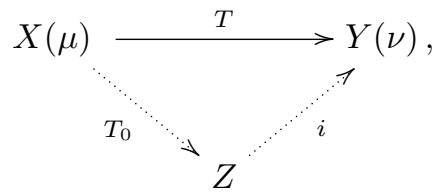

where $Z$ is the smallest B.f.s. in a certain class and $i$ is an inclusion map.

In this setting, our results can give the conditions under which it is possible to find what we call an optimal factorization for such an operator, i.e. to find a factorization diagram as

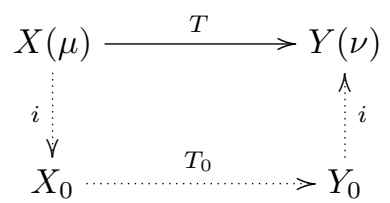

that extends in the left hand side to the biggest space in a class of B.f.s. and restricts the range to the smallest B.f.s. in other class of spaces.

Assume that $X(\mu)$ and $(Y(\mu))^{\prime}$ are order continuous. Consider an operator $T: X(\mu) \rightarrow Y(\mu)$ and define the bilinear map $\mathcal{B}_{T}: X(\mu) \times(Y(\mu))^{\prime} \rightarrow \mathbb{R}$ by $\mathcal{B}_{T}\left(f, y^{\prime}\right)=\left\langle T(f), y^{\prime}\right\rangle, f \in X(\mu), y^{\prime} \in(Y(\mu))^{\prime}$. Take also the vector measures $m_{T}(A)=T\left(\chi_{A}\right) \in Y(\mu)$ and $m_{T^{\prime}}(C)=T^{\prime}\left(\chi_{C}\right) \in(X(\mu))^{\prime}$, being $T^{\prime}$ the adjoint map of $T$. The construction in Section 3 provides two factorizations for $\mathcal{B}_{T}$ through $L^{1}\left(m_{1}\right) \times L^{1}\left(\widetilde{m}_{2}\right)$ and $L^{1}\left(\widetilde{m}_{1}\right) \times L^{1}\left(m_{2}\right)$, respectively. For the first one, in our case it can be easily seen that $m_{1}: \Sigma \rightarrow L\left((Y(\mu))^{\prime}, \mathbb{R}\right)=(Y(\mu))^{\prime \prime}$ is given by $m_{1}(A)=$ $m_{T}(A)=T\left(\chi_{A}\right) \in Y(\mu) \subseteq(Y(\mu))^{\prime \prime}$, and $\tilde{m}_{2}(C):=\left(I_{m_{T}}\right)^{\prime}\left(\chi_{C}\right) \in\left(L^{1}\left(m_{T}\right)\right)^{\prime}$.

This provides, under the adequate separation assumption for the vector measures, the following factorization for $T^{\prime}$.

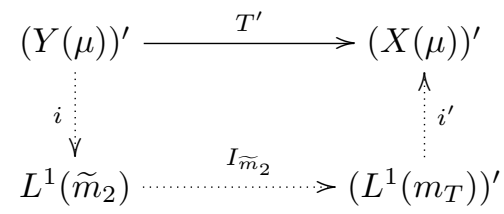


For the second factorization of the bilinear form, notice that $m_{2}: \Sigma \rightarrow L(X(\mu), \mathbb{R})=$ $(X(\mu))^{\prime}$ is given by $m_{2}(C):=T^{\prime}\left(\chi_{C}\right)$, and $\widetilde{m}_{1}: \Sigma \rightarrow L\left(L^{1}\left(m_{T^{\prime}}\right), \mathbb{R}\right)=\left(L^{1}\left(m_{T^{\prime}}\right)\right)^{\prime}$ is defined by $\widetilde{m}_{1}(A):=\left(I_{m_{T^{\prime}}}\right)^{\prime}\left(\chi_{A}\right)$. This gives the diagram

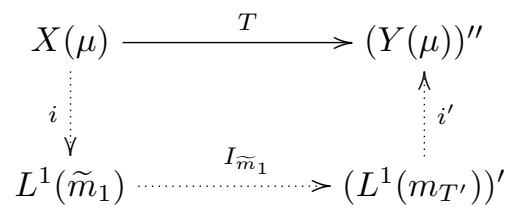

Notice that for each couple of measurable sets $A$ and $C$,

$$
\left\langle m_{1}(A), \chi_{C}\right\rangle=\left\langle T\left(\chi_{A}\right), \chi_{C}\right\rangle=\left\langle\chi_{A}, T^{\prime}\left(\chi_{C}\right)\right\rangle=\left\langle\chi_{A},\left(I_{m_{T}}\right)^{\prime}\left(\chi_{C}\right)\right\rangle=\left\langle\chi_{A}, \widetilde{m}_{2}(C)\right\rangle
$$

and also

$$
\left\langle\widetilde{m}_{1}(A), \chi_{C}\right\rangle=\left\langle\chi_{A}, T^{\prime}\left(\chi_{C}\right)\right\rangle=\left\langle T\left(\chi_{A}\right), \chi_{C}\right\rangle=\left\langle\chi_{A}, m_{2}(C)\right\rangle,
$$

where the duality is computed in the corresponding spaces. These equalities give in fact a rule for computing $\widetilde{m}_{1}$ and $\widetilde{m}_{2}$, which together with the factorizations given above provides two canonical factorization schemes for $T$. Notice also that, after Theorem 3.4 the equivalent conditions given by the possibility of extending $\mathcal{B}_{T}$ to $L^{1}\left(m_{T}\right) \times L^{1}\left(m_{T^{\prime}}\right)$, the coincidence of the spaces $L^{1}\left(m_{1}\right)=L^{1}\left(\widetilde{m}_{1}\right)$ and $L^{1}\left(m_{2}\right)=L^{1}\left(\widetilde{m}_{2}\right)$, and the coincidence of $\mathcal{B}_{12}$ and $\mathcal{B}_{21}$, implies the factorization of $T$ as

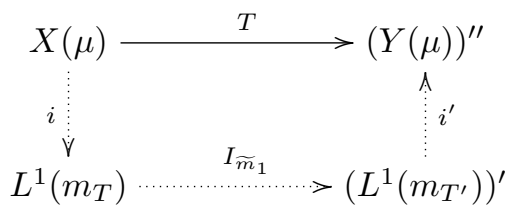

that holds when $T$ is an isomorphism.

\section{REFERENCES}

[1] Calabuig, J.M., Galaz-Fontes, F., Jiménez Fernández, E. and Sánchez Pérez, E.A., Strong factorization of operators on spaces of vector measure integrable functions and unconditional convergence of series. Math. Z. 257 (2007) 381-402.

[2] Calabuig, J.M., Delgado, O. and Sánchez Pérez, E.A., Factorizing operators on Banach function spaces through spaces of multiplication operators. J. Math. Anal. Appl. 364 (2010) 88-103.

[3] Curbera, G.P., Operators into $L^{1}$ of a vector measure and applications to Banach lattices, Math. Ann. 293 (1992) 317-330.

[4] Curbera, G.P. and Ricker, W.J., Optimal domains for kernel operators via interpolation, Math. Nachr., 244 (2002) 47-63.

[5] Curbera, G.P. and Ricker, W.J., Optimal domains for the kernel operator associated with Sobolev's inequality, Studia Math., 158(2) (2003) 131-152 (see also Corrigenda in the same journal , 170 (2005) 217-218).

[6] Delgado, O., Banach function subspaces of $L^{1}$ of a vector measure and related Orlicz spaces, Indag. Math. (N. S.) 15 (2004) 485-495. 
[7] Delgado, O., Optimal domains for kernel operators on $[0, \infty) \times[0, \infty)$, Studia Math. 174 (2006) 131-145.

[8] Delgado, O. and Soria, J., Optimal domain for the Hardy operator, J. Funct. Anal. 244 (2007), 119-133.

[9] Diestel, J. and Uhl, J.J., Vector Measures, Math. Surveys, vol. 15, Amer. Math. Soc., Providence, RI, 1977.

[10] Lindenstrauss, J. and Tzafriri, L., Classical Banach Spaces II, Springer, Berlin, 1979.

[11] Galdames, O. and Sánchez Pérez, E.A., Optimal range theorems for operators with p-th power factorable adjoints, Banach J of Math. Anal. 6(1) (2012), 61-73.

[12] Okada, S., Ricker, W.J. and Sánchez Pérez, E.A. Optimal Domain and Integral Extension of Operators Acting in Function Spaces, Oper. Theory Adv. Math. Appl., vol. 180. Birkäuser, Basel 2008.

Instituto Universitario de Matemática Pura y Aplicada, Universidad Politécnica de Valencia, Camino de Vera s/n, 46022 Valencia, Spain

E-mail address: jmcalabu@mat.upv.es

Centro de Investigación en Matemáticas, Jalisco S/N, Col. Valenciana, CP: 36240 Guanajuato, Gto, México

E-mail address: maite@cimat.mx

Centro de Investigación en Matemáticas, Jalisco S/N, Col. Valenciana, CP: 36240 Guanajuato, Gto, México

E-mail address: galaz@cimat.mx

Instituto Universitario de Matemática Pura y Aplicada, Universidad Politécnica de Valencia, Camino de Vera s/n, 46022 Valencia, Spain

E-mail address: easancpe@mat.upv.es 